\title{
EL INCREMENTO DE LA POBLACIÓN URBANA EN LOS ESTADOS DEL MAGREB CENTRAL
}

\author{
Raquel Barras ${ }^{1}$ \\ Investigadora UNISCI
}

\begin{abstract}
Resumen:
Este artículo analiza el proceso de transición demográfica en el Magreb, en particular la fuerte urbanización que han sufrido tres países: Argelia, Marruecos y Túnez. La población urbana se ha incrementado en la región, dando lugar a múltiples oportunidades, pero también a numerosos desafíos. La aparición de importantes centros urbanos espontáneos entorno a las grandes ciudades como consecuencia del éxodo rural ponen de manifiesto la existencia de notables dificultades demográficas que condicionarán el futuro de los tres países analizados, en el ámbito económico, social y de seguridad. El incremento de la población urbana jugará un papel fundamental en la demanda de alimentos en estos Estados.
\end{abstract}

Palabras clave: Hábitat, urbanización, crecimiento de la población urbana, población rural, suburbios, demanda de alimentos.

Title in English: "Increase of Urban Population in the Central Maghreb Countries"

\section{Abstract:}

This article analyzes the process of demographic transition in the Maghreb, in particular the strong urbanization increase in the three countries: Algeria, Morocco and Tunisia. The urban population has increased in the region, giving rise to many opportunities but also to many challenges. The creation of spontaneous major urban centres around large cities as a result of rural exodus, reveals important demographic challenges that will determine the future of the three countries in the economic, social and security domain. The increasing urban population plays an essential role in the food demand in all these countries.

Keywords: Habitat, urbanization, population growth, urban population, rural population, slums, food demand.

Copyright @ UNISCI, 2013.

Las opiniones expresadas en estos artículos son propias de sus autores, y no reflejan necesariamente la opinión de UNISCI. The views expressed in these articles are those of the authors, and do not necessarily reflect the views of UNISCI

\footnotetext{
${ }^{1}$ Raquel Barras Tejudo es investigadora junior de UNISCI y miembro del Foro Hispano-Argelino. E-mail: raqueltejudo@yahoo.es. 


\section{La distribución espacial de la población: hábitat}

El Magreb es un concepto geográfico que abarca realidades diversas y presenta rupturas en los ámbitos económico, político y geográfico. Tanto la orografía como el clima influyen directamente en la localización de la población y de la actividad económica. En este sentido, la economía de los países se basa en la propia distribución espacial y geográfica.

La incidencia de la orografía y el hábitat en la actividad económica, presenta por un lado, países como Argelia, con rentas del petróleo, y por otro, países más diversificados como Marruecos o Túnez, en los que se conjuga agricultura de exportación, industria y turismo ${ }^{2}$. En esta región el elemento unificador es la geografía puesto que condiciona la agricultura.

\section{Gráfico 1. Distribución espacial de la actividad económica en el Magreb}

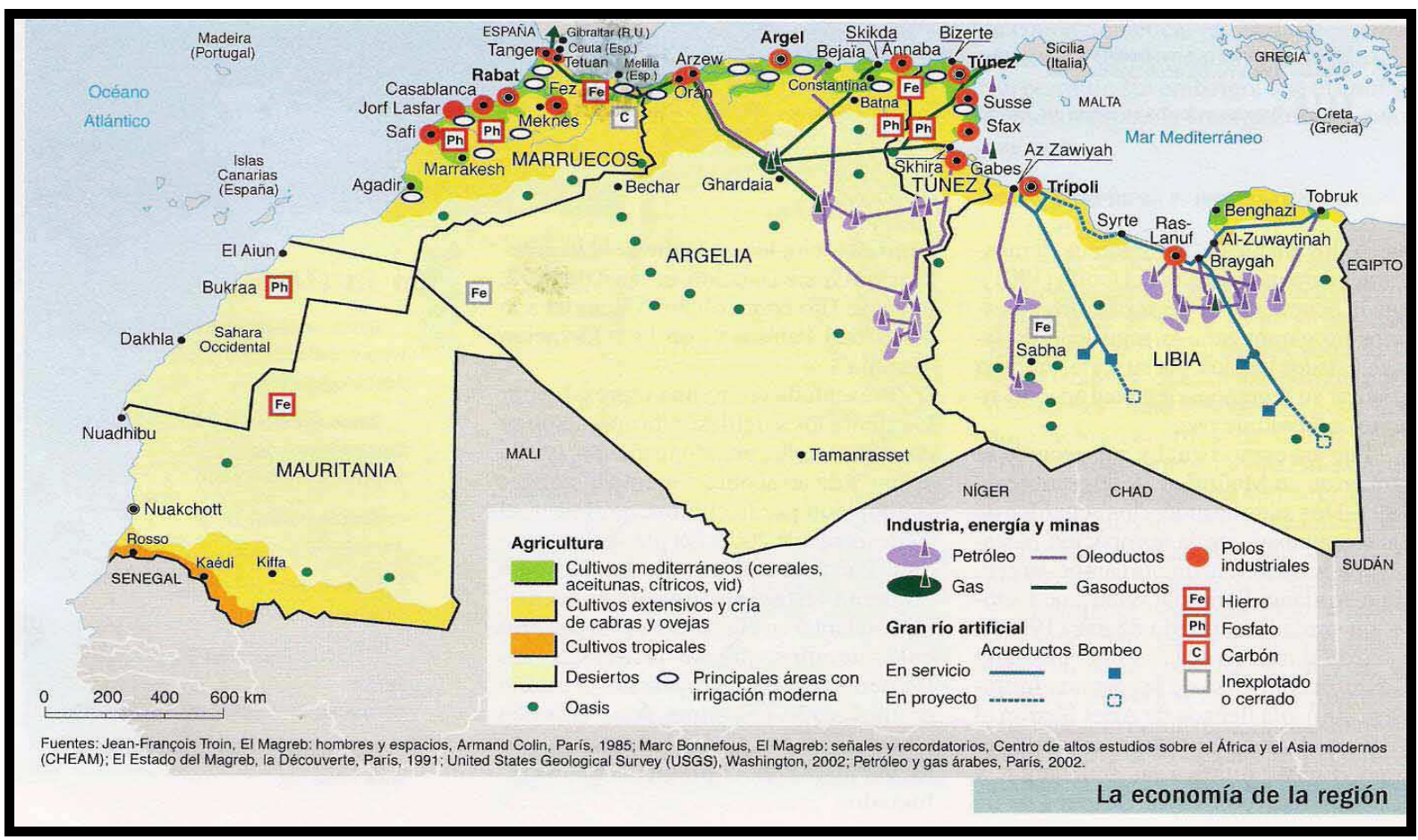

Fuentes: CHEAM, USGS

Del mismo modo, la actividad agrícola se desarrolla en su mayor parte en las franjas costeras, donde las tierras son fértiles y las precipitaciones, permiten el desarrollo de la agricultura mediterránea. Consecuentemente la población tiende a situarse en el litoral, dejando despobladas las regiones interiores y montañosas.

\footnotetext{
${ }^{2}$ Lorca Alejandro, Escribano Gonzalo,: “La situación económica del Magreb”, Real Instituto Elcano, Documento de Trabajo, $\mathrm{n}^{\mathrm{o}} 57$ (Noviembre 2004), en http://www.realinstitutoelcano.org/wps/portal/rielcano/contenido?WCM_GLOBAL_CONTEXT=/elcano/elcano es/zonas es/mediterraneo+y+mundo+arabe/dt57-2004.
} 
La distribución poblacional en la región presenta un determinado hábitat humano ${ }^{3}$. Este concepto, utilizado en la ecología humana y el urbanismo, es una analogía del concepto ecológico de hábitat (ambiente que ocupa una población biológica). Así, el hábitat humano tiene en cuenta el conjunto de factores tanto materiales como institucionales que condicionan la existencia de una población humana localizada. La clasificación y la jerarquización difieren ampliamente de un país a otro, en función de las dimensiones, de las explotaciones, así como del ecosistema, e incluso de las comunicaciones. Cabe destacar que este concepto se ha readaptado en función de los cambios acaecidos en las poblaciones, en los asentamientos (viviendas, establecimientos, y comunidades) y en la propia estructura social. El término proviene de la ecología, e incluye muchas características correlacionadas, especialmente el ecosistema, el ambiente urbano o el ambiente social.

El Magreb padece un doble desequilibrio territorial: entre el litoral y el interior, y entre el campo y la ciudad. El período colonial exacerbó este desequilibrio, al localizar las industrias en las zonas urbanas costeras dotadas de infraestructuras de transporte. Por el contrario, durante el periodo de independencia, la agricultura constituyó uno de los motores del cambio, y se erigió como una de las 'banderas' de la independencia. En este sentido en Túnez se dieron experiencias socializantes y cooperativas basadas en la agricultura y el mundo rural; en Argelia se produjo una autogestión agrícola inicial, que posteriormente se tradujo en una fuerte industrialización del campo; en Marruecos la agricultura también fue un motor del cambio, y una parte esencial del Estado, que llevó a cabo grandes proyectos hidráulicos ${ }^{4}$, así como la promoción y el desarrollo de cultivos de exportación (agrios, patatas y tomates) y una colonización de tierras concebida como un sustitutivo de la reforma agraria ${ }^{5}$.

Actualmente la actividad rural ya no está tan centrada en la agricultura. El medio rural ha experimentado una rápida diversificación en el que el componente agrícola se encuentra en una fase de adaptación a planteamientos neoliberales, la iniciativa privada tanto nacional como extranjera tiene una influencia creciente en el sector en Marruecos y Túnez, y donde se ha llevado a cabo un intenso programa de reprivatización de las explotaciones, convirtiendo las zonas rurales en sociedades más diversificadas.

\section{Población rural y urbana en el Magreb}

En muchos países en desarrollo, el crecimiento de la población ha sido un factor importante para el rápido crecimiento de las mega-ciudades y para los suburbios informales. Los últimos aumentos de la población y el crecimiento proyectado de la misma, son cruciales para el actual incremento de la demanda de alimentos en la región.

En este sentido los países del Magreb deberán afrontar en los próximos años desafíos de diversa índole como consecuencia de su situación demográfica.

La región ofrece países desigualmente poblados; los tres países objeto de estudio: Marruecos, Argelia y Túnez son los países donde viven mayor número de personas superando

\footnotetext{
3 En: UNESCO: "Las ciudades y los hábitats humanos", Oficina de Información Pública, en http://www.unesco.org/bpi/pdf/memobpi51_towns_es.pdf.

${ }^{4}$ El país llevó a cabo una amplia y ambiciosa política de embalses.

${ }^{5}$ Lenormand P.: "Transformaciones territoriales y cambios sociales en los campos magrebíes", Ería: Revista cuatrimestral de geografía, no 38 (1995), pp. 217-234.
} 
los 80 millones de personas ${ }^{6}$. En comparación con otros países de la región como Mauritania o Libia, las mayores densidades demográficas, superiores a los 60 habitantes por $\mathrm{km}^{2}$, se dan en Marruecos y en Túnez.

Según estimaciones para el año 2012, Marruecos cuenta con 32,3 millones de personas, con una densidad de población de 70,5 hab. $/ \mathrm{km}^{2}$ (43,05 hab. $/ \mathrm{km}^{2}$ incluyendo el Sahara Occidental), mientras que en Argelia son 37,4 millones de personas, siendo su densidad de población de 13,8 hab. $/ \mathrm{km}^{2}$. Del mismo modo, hay 10,7 millones de tunecinos, con una densidad poblacional de $61,7 \mathrm{hab} . / \mathrm{km}^{2}$.

La transición demográfica en los países del Magreb pone de manifiesto la influencia de la política, la economía y la sociología en la composición de la población y de la familia. La demografía del Magreb ha experimentado una revolución en los últimos años. Sin embargo, tanto la natalidad, como la nupcialidad o la mortalidad en esta región no son propias aún de los países desarrollados.

Los comportamientos poblacionales vienen diferenciados por regiones, en particular afectados por el medio de residencia, urbano o rural, así como por los segmentos de población, el nivel socioeconómico, y el nivel de educación.

Las principales magnitudes poblacionales del Magreb $^{7}$ han sufrido grandes transformaciones en las últimas décadas, influyendo de forma directa en la distribución espacial de la población. En este sentido, la población rural y urbana ha evolucionado desde los años cincuenta en Marruecos, Túnez y Argelia. Tradicionalmente la población rural había sido predominante, sin embargo, los tres países han protagonizado cómo, entre los años ochenta y noventa, se invierte la población rural y urbana, y, por primera vez, la población urbana supera a la población rural.

Entre 1980 y 1990 se produce un fuerte proceso de urbanización causado principalmente por el boom de la emigración del campo a la ciudad, la falta de perspectivas de empleo en el campo, el atractivo de las ciudades, por la estructura de la economía y el grado de desarrollo económico ${ }^{8}$. El momento de cambio se sitúa en Marruecos y en Argelia en los años noventa, mientras que en Túnez se da una década antes, en 1980.

\subsection{Marruecos:}

En Marruecos la población rural en los años cincuenta superaba el 70\%, mientras que en la actualidad, se sitúa ligeramente por encima del $40 \%$, esto es más de 32 millones de personas. En el año 2001, de un total de 30 millones de habitantes, el país contaba con un $56 \%$ de población urbana, mientras que la población de los suburbios de las grandes ciudades alcanzaba el $33 \%$ de la población ${ }^{9}$.

\footnotetext{
6 Datos estimados a julio de 2012; fuente: CIA en https://www.cia.gov/library/publications/the-worldfactbook/index.html

${ }^{7}$ Para más información ver el capítulo de Gracia Abad en este mismo número de la revista.

8 Brauch Hans Günter: "Urbanization and Natural Disasters in the Mediterranean Population Growth and Climate Change in the 21st Century. Case Studies on Izmir, Algiers and Alexandria", Urban Research, en http://urban-research.blogspot.com.es/2012/04/urbanization-and-natural-disasters-in.html

${ }^{9}$ Datos 2001, estimaciones: UN-HABITAT, en:

http://www.unhabitat.org/content.asp?typeid=19\&catid=555\&cid=5374.
} 


\section{Gráfico 2. Población rural y urbana de Marruecos}
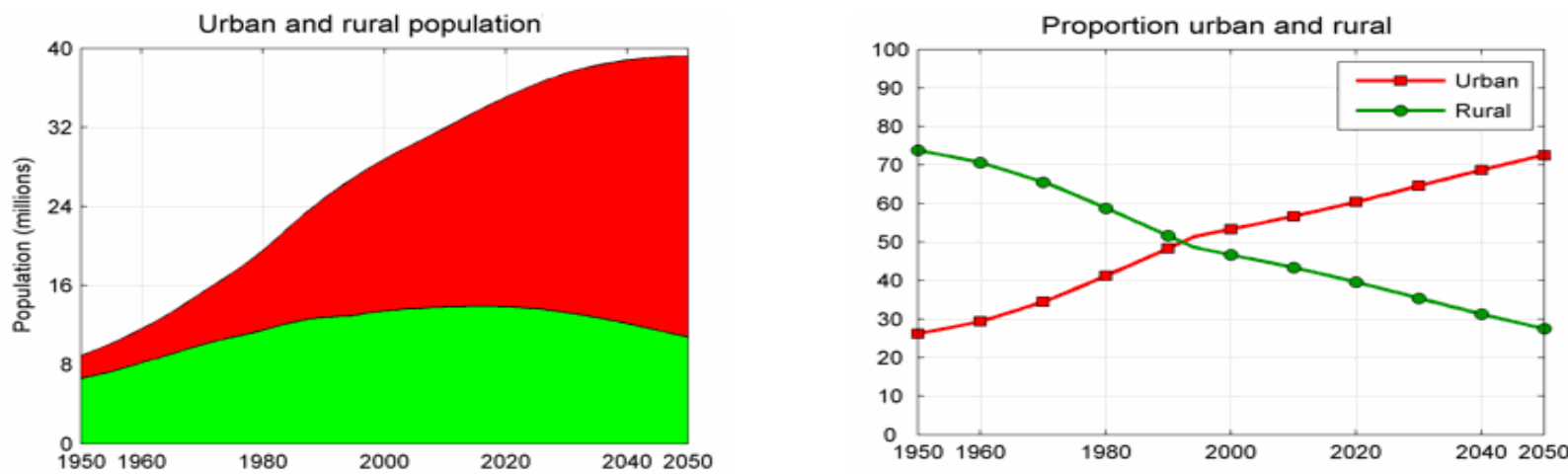

Fuente: United Nations Population Division, Country Profiles ${ }^{10}$.

La tendencia para el año 2050, es que la población rural del país sea inferior al 30\%, por encima de los 6 millones de personas. No se prevé que las poblaciones rurales disminuyan en Marruecos en el periodo analizado de cien años (1950-2050), sino que se incrementarán ligeramente. Esto es debido principalmente a la elevada tasa de natalidad en el mundo rural, que si bien 'envía' mucha población a los centros urbanos, 'absorbe' buena parte de su propia natalidad.

\subsection{Argelia:}

En Argelia la evolución de la población urbana y rural ha sido similar a la de Marruecos. El país rozaba en los años cincuenta el $80 \%$ de la población rural, llegando en la actualidad a invertirse completamente. En el año 2001, de un total de 31 millones, el 58\% correspondía a población urbana, mientras que la población que se asentaba en los suburbios de las grandes ciudades era el $12 \%{ }^{11}$.

\section{Gráfico 3. Población rural y urbana de Argelia}
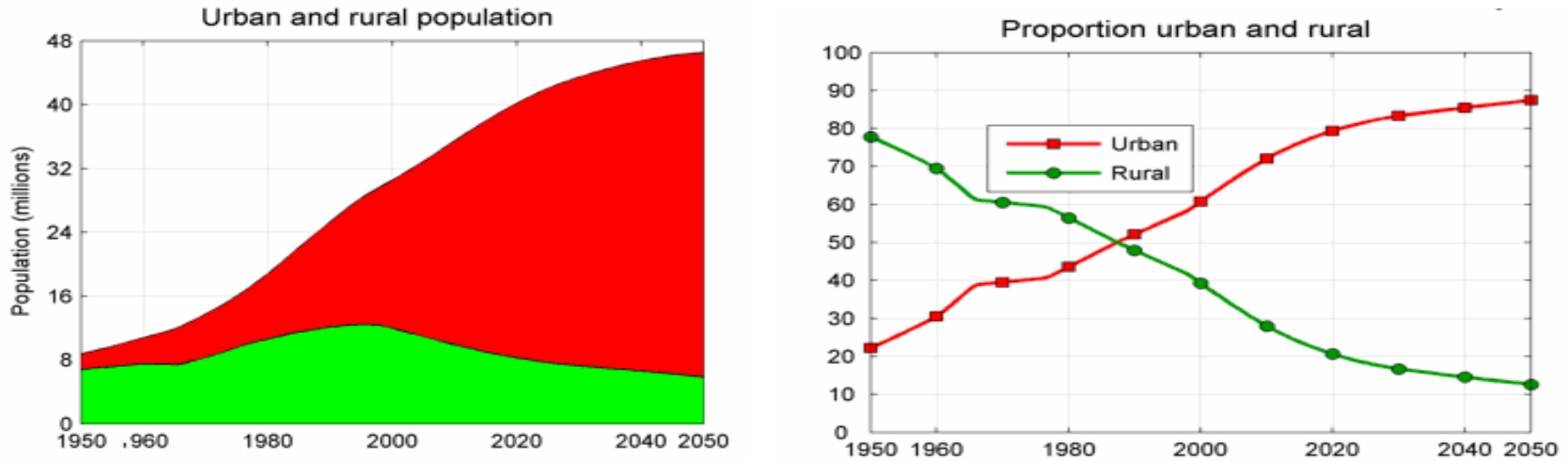

Fuente: United Nations Population Division, Country Profiles ${ }^{12}$.

\footnotetext{
10 "World Urbanization Prospects: The 2011 Revision", United Nations (UN), Department of Economic and Social Affairs, Population Division New York (2012), en http://esa.un.org/unpd/wup/Country-Profiles/countryprofiles 1.htm.

${ }^{11}$ UN-HABITAT, op. cit.

${ }^{12}$ World Urbanization Prospects, op. cit.
} 
Actualmente la población urbana argelina supera el 70\%, alrededor de los 35 millones de habitantes. En el año 2020 alcanzará el 80\%, y se estima que para el año 2050, rozará el 90\% de la población, siendo casi 48 millones de habitantes en áreas urbanas. Cabe destacar que en Argelia el proceso de urbanización será más agudo e intenso, puesto que la variación se produce de extremo a extremo.

\subsection{Túnez:}

En 1950 la población rural de Túnez prácticamente era del 70\%. En 1980 se produce el cambio, y la población rural y urbana alcanzan niveles similares, al 50\%. En el año 2001, la población urbana en Túnez era del $66 \%$, mientras que el porcentaje de personas que se asentaron en los suburbios de las grandes ciudades fue del $4 \%$, de un total de 10 millones de personas $^{13}$. Actualmente los tunecinos que viven en áreas urbanas casi alcanzan el $70 \%$ de la población, superando los 10 millones de personas. Para el año 2050, la previsión es que la población urbana alcance el $80 \%$, por encima de los 12 millones de habitantes, frente a los 2 millones que poblarán las áreas rurales.

\section{Gráfico 4. Población rural y urbana en Túnez}
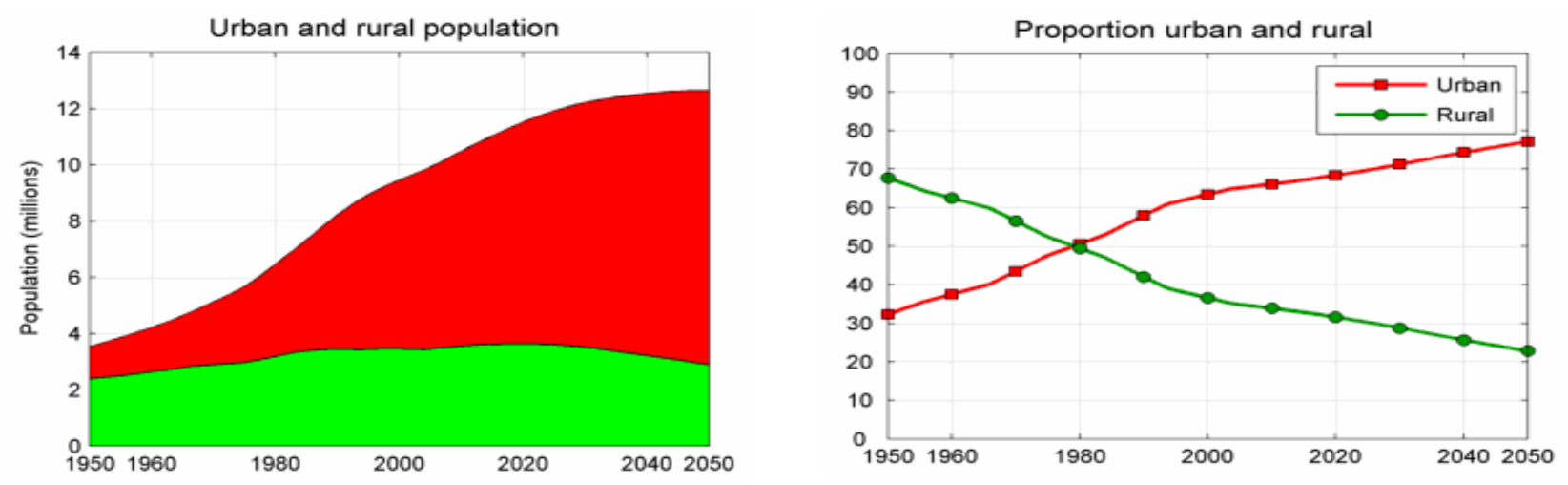

Fuente: United Nations Population Division, Country Profiles ${ }^{14}$.

\section{Diferencias entre el campo y la ciudad}

\subsection{Principales magnitudes: campo vs ciudad}

A la hora de abordar la población rural en el Magreb, es necesario observar la incidencia de la tasa de natalidad, que muestra una tendencia de crecimiento, tal y como se explica en esta revista $^{15}$, arroja una población eminentemente joven, lo que afecta directamente a la población rural, debido a un mayor crecimiento de la población en las áreas rurales en comparación con las zonas urbanas.

Del mismo modo, otro indicador relevante es la esperanza de vida, que está directamente relacionada con las mejoras socio-sanitarias en los países de la región. En particular, la cobertura sanitaria en el Magreb se ha construido de forma rápida a través de una

\footnotetext{
${ }^{13}$ UN-HABITAT, op. cit.

${ }^{14}$ World Urbanization Prospects, op. cit.

${ }^{15}$ Artículo de la profesora Gracia Abad.
} 
amplia red de profesionales de la enfermería que han permitido mejorar la situación sanitaria general y el número de habitantes por médico ha disminuido notablemente, principalmente en las zonas urbanas (si bien sigue siendo diez veces superior al que se registra en nuestro país $)^{16}$.

Otro factor a tener en cuenta es la educación de la mujer, que ha supuesto una auténtica revolución en la concepción de la familia ${ }^{17}$. El abandono progresivo de la familia numerosa a favor de la familia reducida se ha producido con diferente intensidad en países y regiones, si bien una de las causas comunes ha sido el profundo cambio del papel de la mujer en la familia $\mathrm{y}$ en la sociedad.

Por un lado, el aumento del nivel de la escolarización de la mujer, demuestra una estrecha relación entre escolarización y fecundidad ${ }^{18}$, a mayor nivel educativo, menor fecundidad, puesto que la salida de la mujer del hogar paterno rompe con la familia tradicional. Así, las mujeres con instrucción básica, secundaria o superior tienen la pauta de fecundidad próxima a las mujeres de la orilla norte del Mediterráneo (2,1 hijos), mientras que las mujeres analfabetas conservan inalterada la fecundidad no planificada. La tasa de alfabetización es menor en las zonas rurales, y se sitúa en Marruecos en el 56,1\%, en el 69,9\% en Argelia y en el 74,3\% en Túnez.

Por otro, el cambio del papel que la mujer debe desempeñar en la sociedad va a permitir y contribuir a una nueva perspectiva demográfica. La introducción de la mujer en el trabajo, principalmente en el medio urbano; el nivel de actividad femenina entre 20 y 49 años es el del 25,7\% en Túnez, del 23,7\% en Marruecos y del 17,5\% en Argelia. Si bien la región en su conjunto se sitúa a nivel mundial en un índice relativamente bajo en relación con las mujeres trabajadoras en las ciudades, es importante destacar que para estos países en sí, ha supuesto verdaderamente un gran cambio, alterando los pilares básicos del modelo de organización familiar patriarcal, erosionando el papel estrictamente de madre y esposa de la mujer, que ha ido adquiriendo una mayor presencia pública en la vida económica.

Esto ha contribuido a un cambio en las relaciones de género, a la modificación del status privado y público de las mujeres, a un mayor empoderamiento, junto con modificaciones en la legislación y código de familia. En cifras en el Magreb encontramos un 20\% de mujeres funcionarias, o una representación política femenina que alcanza el 15\%. En algunas zonas del mundo rural las mujeres son el motor del cambio, y han propiciado cambios normativos, así como creado un fuerte movimiento asociacionista. Destacar del mismo modo el papel y la influencia de las políticas de igualdad introducidas por la cooperación internacional en la región.

Otros factores a tener en cuenta han sido los cambios que se han producido en los países del Magreb respecto a la poligamia y al repudio y cómo estos han influido en el matrimonio reflejándose en la estructura misma de las sociedades.

La política de los Estados ha tenido una influencia indirecta en la población por medio de las políticas de familia. Los elevados costes que suponen para los países el crecimiento

\footnotetext{
${ }^{16}$ Lenormand, op. cit., nota 4.

${ }^{17}$ Fargues Philippe (1996): "La demografía en el Magreb: una clave para comprender la política” pp. 129-142, en M. A., Roque (coord.): Las culturas del Magreb: antropología, historia y sociedad, L'Institut Català de la Mediterrània d'Estudis i Cooperació.

${ }^{18}$ Mohammed, Arkoun et al. (ed.) (1996): Las Culturas Del Magreb: Antropología, Historia y Sociedad, L'Institut Català de la Mediterrània d'Estudis i Cooperació, pp. 132-133.
} 
demográfico acelerado, han condicionado la política de familia. Así en Marruecos y Túnez, países con economías de producción diversificada, han experimentado cierto descenso de la fecundidad debido a la necesidad de movilización de la fuerza de trabajo familiar, incluida la femenina. Por el contrario, en los países del Golfo, con economías de redistribución por rentas del petróleo, desde los años setenta se han venido estimulando los nacimientos, a través de ciertas medidas de apoyo y ayuda a las familias. Argelia se sitúa a medias de los dos grupos, puesto que, a pesar de las rentas por hidrocarburos, al contar con una población tan numerosa, no permite el sistema de reparto que impera en los países del Golfo ${ }^{19}$.

La pobreza afecta al $25 \%$ de la población rural frente al $12 \%$ de la población urbana. No obstante en el Magreb actual se han consolidado áreas de chabolismo y miseria en los suburbios de las ciudades. En este sentido, cabe destacar que la pobreza rural no ha generado tensiones ni ha llevado a socavar la cohesión social, mientras que esta realidad en las ciudades supone un caldo de cultivo para resentimientos y radicalismos desestabilizadores.

\subsection{Localización y servicios}

La localización está muy ligada a la existencia de una mayor oferta de servicios. El trabajo, los ingresos, servicios públicos, escuelas y espacios verdes, existen en función del 'sitio'. Las cuidadas zonas residenciales de las afueras, las ciudades punteras, los centros comerciales y de negocios, los guetos urbanos, las zonas industriales desocupadas y los parques de oficinas, todo forma parte de un complejo entramado urbano del que dependen las oportunidades ${ }^{20}$.

La población rural se ve afectada por un paulatino deterioro de las condiciones de vida, en comparación con la evolución de la calidad de vida en las áreas urbanas. No obstante el masivo éxodo rural $^{21}$, es necesario precisar que no es que las poblaciones rurales hayan desaparecido o disminuido significativamente, a semejanza de lo ocurrido en España en los años sesenta, sino que se ha producido una fuerte tendencia de concentración de las mismas.

Tabla 1. Diferencia de indicadores entre la población rural y urbana

\begin{tabular}{|c|c|c|c|c|}
\hline \multirow{2}{*}{$\%$} & \multicolumn{2}{|c|}{ Acceso agua potable } & \multicolumn{2}{c|}{ Acceso Sanidad } \\
\hline & URBANA & RURAL & URBANA & RURAL \\
\hline Argelia & 98 & 79 & 90 & 88 \\
\hline Marruecos & 100 & 60 & 94 & 52 \\
\hline Túnez & 100 & 84 & 96 & 64 \\
\hline
\end{tabular}

Fuente: Naciones Unidas ${ }^{22} /$ Año 2008

\footnotetext{
${ }^{19}$ Fargues, op. cit. nota 16, pp. 119-131.

${ }^{20}$ Savitch, Hank V.: "Las novedades de la mundialización y sus repercusiones en las ciudades". Revista Internacional de Ciencias Sociales, $\mathrm{n}^{\circ} 172$ (2002), en http://www.unesco.org/new/fileadmin/MULTIMEDIA/HQ/SHS/pdf/172-Fulltext172spa.pdf.

${ }^{21}$ Para más información véase el artículo de Gloria Inés Ospina en esta misma revista.

${ }^{22}$ Datos para 2001 en: http://esa.un.org/unpd/wup/Country-Profiles/country-profiles_1.htm y para 2008 en: http://www.unhabitat.org/downloads/docs/GRHS2011_Full.pdf.
} 
Así, en Marruecos, los zocos rurales han servido de germen de urbanización en las poblaciones circundantes. Del mismo modo ha ocurrido en Argelia, con lo que se han denominado los 'pueblos de la colonización', y que han contribuido indudablemente a reducir el hábitat disperso en este país. En Túnez del mismo modo, la población rural diseminada está dejando de ser predominante en la mayoría de las provincias. A pesar de los avances, existe aún una diferencia considerable de acceso a los servicios en los centros urbanos respecto de los municipios rurales.

Las grandes diferencias de oferta de servicios entre el campo y la ciudad en los países del Magreb suponen aún hoy una diferencia nada desdeñable, muy alejada de la realidad de los países desarrollados.

\section{Procesos de urbanización}

En 1960 los niveles de urbanización en el Magreb no superaban en ningún caso el 25\%, oscilando entre el 3,4\% en Mauritania y el 36\% en Túnez. En 1995, el ritmo de urbanización variaba dependiendo de los países. La población del Magreb no sólo ha crecido rápidamente, sino que también se ha ido urbanizando. El exponencial crecimiento de las poblaciones urbanas ha sido causado por el éxodo rural. Las malas condiciones de vida en el medio rural han forzado el éxodo hacia las urbes, cuyos servicios se han visto desbordados por el aluvión de emigrantes rurales y el dinamismo demográfico.

El proceso de urbanización está directamente relacionado con la evolución de la economía, desde el nacimiento del modo de producción capitalista de la mando de la colonización, junto con la aceleración de dicho proceso durante la política social llevada a cabo tras la independencia, y que culmina con la globalización de la economía mundial. En este sentido el desarrollo de los sectores de la economía de servicios ha contribuido al fuerte fenómeno de urbanización que se da en los países del Magreb.

Tabla 2. Tasas de urbanización

\begin{tabular}{|llllllllll|}
\hline \multicolumn{1}{|c}{$\%$} & 1950 & 1960 & 1970 & 1980 & 1990 & 2000 & 2010 & 2020 & 2030 \\
Argelia & 22.3 & 30.4 & 39.5 & 43.5 & 51.4 & 57.1 & 62.2 & 67.5 & 71.7 \\
Marruecos & 26.2 & 29.2 & 34.6 & 41.3 & 48.4 & 55.5 & 61.7 & 66.7 & 71.0 \\
Túnezz & 31.2 & 36.0 & 44.5 & 51.5 & 57.9 & 65.5 & 71.3 & 75.2 & 78.4 \\
\hline
\end{tabular}

Fuente: Naciones Unidas

Como se ha visto anteriormente los países de la región sufren un fuerte proceso de urbanización entre 1980 y 1990, que alcanza su punto álgido en los tres países alrededor del año 2000. Se estima que en el año 2030 la tasa de urbanización alcance en Argelia y Marruecos el 70\%, mientras que en Túnez será del 78,4\%.

El país menos urbanizado a mediados del siglo XX era Argelia, que contaba con una tasa de urbanización del 22,3\%, seguido por Marruecos, que se situaba en el 26,2\%, y por 
último Túnez, con un 31,2\%. La tasa de urbanización alcanzaba en el año 2000 el 57,1\%, el 55,5\% y el 65,5\% en Argelia, Marruecos y Túnez respectivamente, duplicándose en cincuenta años ${ }^{23}$.

En el año 2001 la tasa del crecimiento anual de la población urbana en Argelia se situaba en el $3 \%^{24}$, mientras que actualmente (2010-2015) se sitúa en el $2,3 \%^{25}$, y se estima que, para la década 2020-2030, se reduzca hasta el $0,58 \%{ }^{26}$. Actualmente la tasa de urbanización se sitúa en Argelia en el 62,2\%.

El Marruecos el crecimiento anual de la población urbana se situaba en el año 2001 en el $3 \%^{27}$; sin embargo en la actualidad ha descendido hasta el $2,1 \%^{28}$ para el periodo 2010 2015 , lo que revela cierto freno en la urbanización del país. Las estimaciones para la década de 2020-2030 suponen un descenso de la tasa de crecimiento de la población urbana, hasta el $0,78 \%$.

En Túnez, la tasa de urbanización se sitúa en el 71,3\%. Actualmente la tasa de crecimiento de urbanización es del 1,5\% (2010-2015), muy alejada del 3\% del año $2001^{29}$. Para la década 2020-2030 el crecimiento de la población urbana se va a reducir en sólo una décima, situándose en el $0,55 \%$.

\subsection{Crecimiento de las poblaciones urbanas}

Mientras algunas ciudades se están haciendo cada vez más pequeñas, muchos centros urbanos están presenciando un crecimiento demográfico rápido y descontrolado, lo que está provocando una urbanización acelerada. Gran parte de este crecimiento ocurre en los países en desarrollo y está concentrado en asentamientos informales y barrios precarios.

Tabla 3. Crecimiento de los principales centros urbanos 1950-2050

\begin{tabular}{|lcccccccc|}
\hline Millones de personas & 1950 & 1960 & 1970 & 1980 & 1990 & 2000 & 2010 & 2015 \\
\hline Argel & 0.50 & 0.81 & 1.28 & 1.62 & 1.91 & 2.76 & 3.74 & 4.14 \\
Túnez & 0.47 & 0.58 & 0.74 & 1.14 & 1.57 & 1.90 & 2.25 & 2.41 \\
Casablanca & 0.63 & 0.97 & 1.51 & 2.11 & 2.69 & 3.36 & 4.22 & 4.61 \\
Rabat & 0.15 & 0.23 & 0.49 & 0.81 & 1.16 & 1.61 & 2.13 & 2.34 \\
Fez & 0.17 & 0.28 & 0.37 & 0.51 & 0.68 & 0.90 & 1.18 & 1.30 \\
Marrakech & 0.21 & 0.24 & 0.32 & 0.42 & 0.58 & 0.82 & 1.10 & 1.21 \\
& & & & & & & & \\
\hline
\end{tabular}

\footnotetext{
${ }^{23}$ Estimaciones Naciones Unidas del año 2002.

${ }^{24}$ En "Afghanistan", United Nations, Department of Economic and Social Affairs, Population Division (25 abril 2005), en http://esa.un.org/unpd/wup/Country-Profiles/country-profiles_1.htm

${ }^{25}$ Datos para 2010-2015; fuente: CIA en https://www.cia.gov/library/publications/the-world-factbook/index.html.

${ }^{26}$ En estimaciones año 2011: en http://www.unhabitat.org/downloads/docs/GRHS2011_Full.pdf.

${ }^{27}$ En "Afghanistan", op. cit.

${ }^{28}$ Datos para 2010-2015; fuente: CIA en https://www.cia.gov/library/publications/the-world-factbook/index.html.

${ }^{29}$ Datos 2001, en "Afghanistan", op. cit.

${ }^{30}$ Estimaciones año 2011: en http://www.unhabitat.org/downloads/docs/GRHS2011_Full.pdf.
} 
Fuente: Naciones Unidas

En los países objeto de estudio, Túnez es la ciudad más poblada del país, con 759.000 habitantes $^{31}$. En Argelia el mayor número de población se concentra en la capital, Argel, con 2,74 millones y Oran, con 770.000 habitantes $^{32}$. En Marruecos, el país menos urbanizado, las ciudades más pobladas son Casablanca, con 3.245.000 habitantes, la capital Rabat, con 1.770.000 habitantes, Fez, con 1.044.000, seguido por Marrakech y Tánger, con 909.000 y 768.000 habitantes respectivamente ${ }^{33}$.

Las principales aglomeraciones urbanas, situadas en el litoral, son: Argel (Argelia) y Casablanca (Marruecos); Túnez (Túnez) y Rabat, que superan los 2 millones de habitantes; y Trípoli (Libia), con alrededor de millón y medio de habitantes. Una minoría privilegiada habita en zonas residenciales, mientras que la mayoría de la población se hacina en barrios degradados de las periferias de las grandes urbes.

El aumento de la urbanización y la economía están intrínsecamente relacionados ${ }^{34}$, principalmente porque las poblaciones urbanas atraen mayor riqueza, son mucho más prósperas y siguen atrayendo a las poblaciones procedentes del campo.

Los siguientes gráficos muestran la población urbana en función del tamaño de las ciudades, elemento clave para entender los retos a los que se enfrentan los centros urbanos poblacionales de la región, en relación con la seguridad alimentaria.

\subsubsection{Argelia}

En Argelia no existe ningún centro urbano que supere los 5 millones de personas. Desde mediados de los años sesenta se han incrementado sustancialmente las poblaciones de entre 1 y 5 millones de personas, inexistentes hasta la fecha. Se aprecia una tenue tendencia entre los años 2000 y 2025 de aparición de centros urbanos de entre 750.000 y 1 millón de habitantes.

\footnotetext{
31 Datos estimados año 2009; fuente: CIA en https://www.cia.gov/library/publications/the-worldfactbook/index.html

${ }^{32}$ Ibid.

${ }^{33}$ Ibid.

${ }^{34}$ Cuando la producción agrícola, minera o maderera alcanza un cierto nivel y pueden llegar al mercado ciertos excedentes, surgen nuevas oportunidades para la conversión de materias primas en nuevos productos. La conversión tiene lugar en zonas urbanas cuya capacidad tecnológica y comercial revierte a las economías rurales, lo que permite todavía más eficiencia y libera más individuos para trabajar en otro ramo. La nueva tecnología y las nuevas inversiones ofrecen todavía más oportunidades y una sinergia sin fisuras entre todos los sectores de producción; en Savitch, op. cit., nota 19.
} 


\section{Gráfico 5. Crecimiento de la población urbana en Argelia}

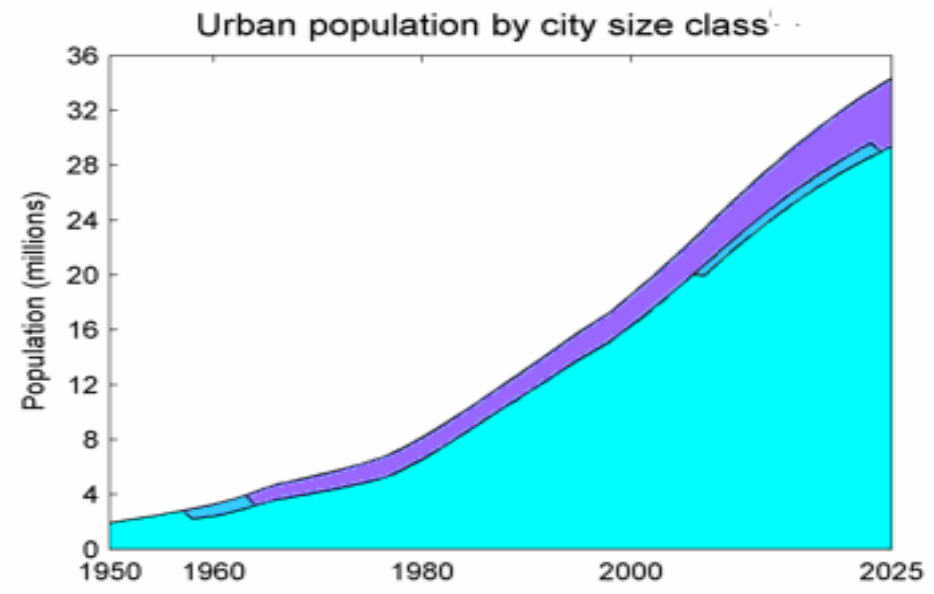

Fuente: United Nations Population Division, Country Profiles ${ }^{35}$

\subsubsection{Marruecos}

Marruecos es el país que muestra los centros urbanos más numerosos de la región, de entre 1 y 5 millones de habitantes. El gráfico incluye las aglomeraciones urbanas con una población inferior a 750.000 habitantes que son una gran mayoría, puesto que incluye todas las poblaciones con carácter residual. Los centros urbanos menos habituales en el país son los que oscilan entre los 750.000 y el millón de habitantes, si bien se estima un incremento irregular de este tipo de ciudades hasta el año 2025.

\section{Gráfico 6. Crecimiento de la población urbana en Marruecos}

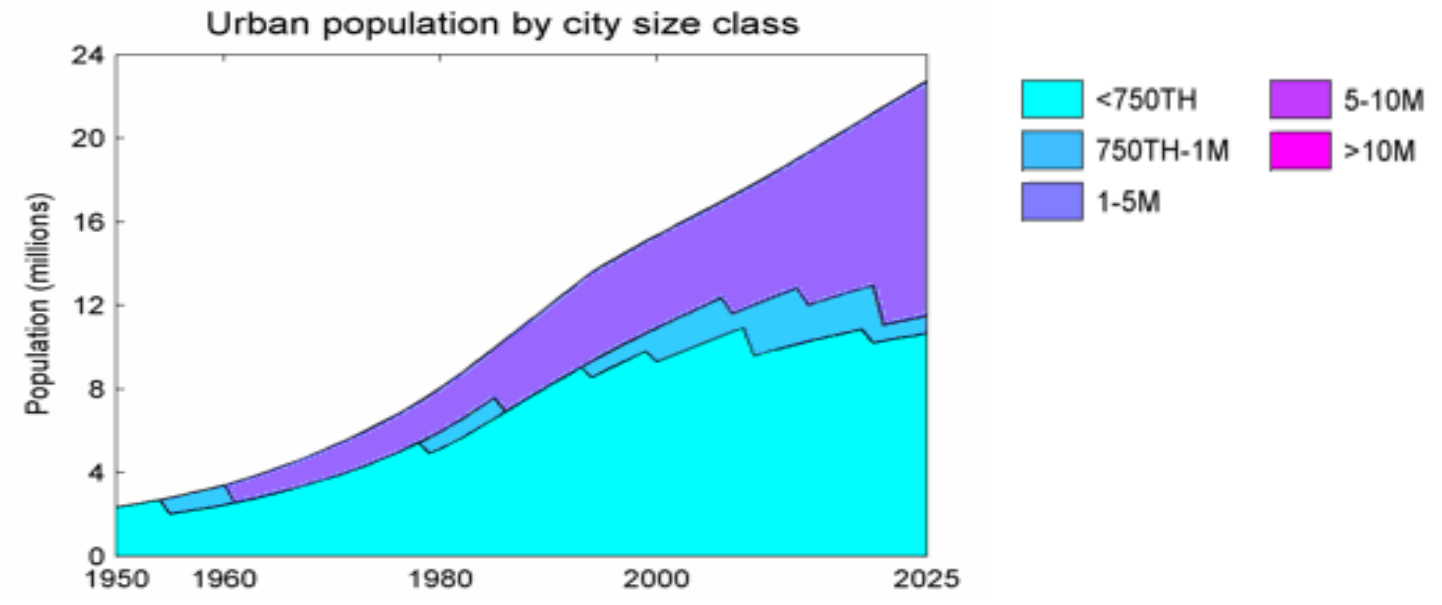

Fuente: United Nations Population Division, Country Profiles ${ }^{36}$

\footnotetext{
${ }^{35}$ En "Afghanistan", op. cit.

36 Ibid.
} 


\subsubsection{Túnez:}

En Túnez los centros urbanos predominantes son aquellos con una población inferior a 750.000 habitantes. Recientemente se han creado focos urbanos con entre 750.000 y 1 millón de personas, y se prevé para el año 2025 el crecimiento de la capital, actualmente el foco urbano más poblado, se sitúe en la franja de entre 1 y 5 millones de habitantes.

\section{Gráfico 7. Crecimiento de la población urbana en Túnez}

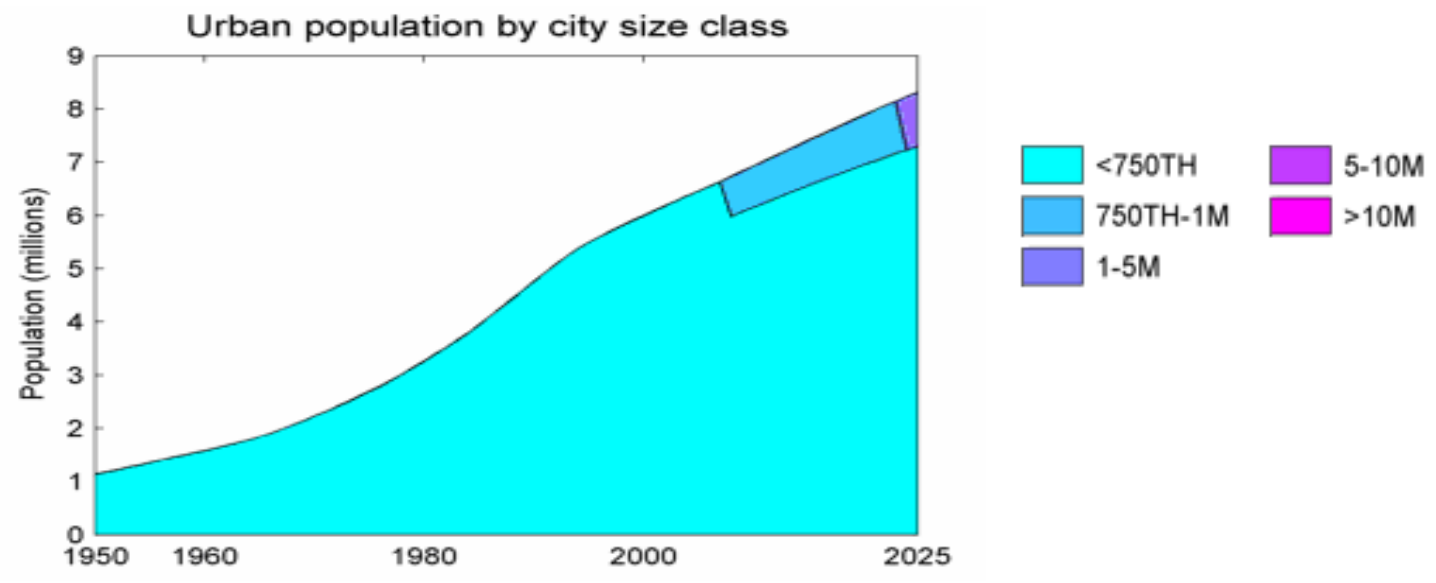

Fuente: United Nations Population Division, Country Profiles ${ }^{37}$

La capital sufrió un rápido crecimiento en las últimas décadas, en particular con un desmesurado crecimiento de nuevas superficies de la periferia, así como de los barrios periféricos espontáneos, olvidando la Medina y el casco histórico de la ciudad ${ }^{38}$.

El proceso de urbanización en los países del Magreb aún no ha culminado, si bien se han llevado a cabo ciertos procesos que han dotado a estos países del de formas políticas más complejas, así como de un mayor desarrollo cultural. El aumento de la urbanización ha sido un proceso paralelo a la mundialización y a la actividad económica. Las zonas metropolitanas de Europa y América del Norte se hicieron más ricas durante la transformación, aunque claramente a medida que el proceso madura, el índice de urbanización se estanca ${ }^{39}$.

\subsection{Desafíos de la gestión urbana: crecimiento de los suburbios}

Los procesos de urbanización de las grandes ciudades magrebíes han supuesto profundos cambios sociales en relación a la movilidad social y a la integración urbana, principalmente porque el acceso a la identidad ciudadana se asienta esencialmente en el éxodo rural como fenómeno irreversible y en la asimilación de dicha población en los propios centros urbanos ${ }^{40}$.

\footnotetext{
${ }^{37}$ Ibid.

${ }^{38}$ Akrout-Yaiche Semia: "La función de los protagonistas locales en la gestión urbana. La experiencia de la ciudad de Túnez", Revista Internacional de Ciencias Sociales, no 172 (2002), en http://www.unesco.org/new/fileadmin/MULTIMEDIA/HQ/SHS/pdf/172-Fulltext172spa.pdf.

${ }^{39}$ Savitch, op. cit., nota 19.

${ }^{40}$ Bouchara Zannad, Traki: "Las nuevas formas de identidad cotidiana en el Magreb: el caso de Túnez". CIDOB (2000), en http://www.cidob.es/Castellano/Publicaciones/Afers/afers.html.
} 
Este proceso no ha sido homogéneo en las ciudades magrebíes, si bien representa ciertas pautas comunes: intensificación del éxodo rural a partir de los años cincuenta; densificación de las ciudades, en particular de la Medina; y cierta ruralización de las ciudades. Este fenómeno se ha denominado ukalización ${ }^{41}$, entendido como la ruralización del espacio doméstico tradicional.

Se observa la creación de barrios interurbanos que han crecido de forma espontánea, un crecimiento que se denomina hábitat espontáneo, que ha dado lugar a lo que se conoce como bidonvilles (chabolas), que se caracteriza por ser barriadas formadas por viviendas provisionales, a menudo insalubres, con escasos servicios públicos y mobiliario urbano degradado, y frecuentemente habitado por personas marginadas socialmente.

Adicionalmente, el casco histórico de las principales ciudades magrebíes, a menudo se ha convertido en áreas urbanas deprimidas y barrios envejecidos $(60 \%$ en el caso de la ciudad de Túnez), donde se hacinan los habitantes llegados de las áreas rurales. Este 'cinturón rojo' supuso una reacción al orden previamente establecido en el ámbito urbanístico.

El hábitat periurbano constituye por tanto una ciudad alternativa, nuevas ciudades o 'barrios de aluvión' que se enfrentan a importantes retos en el ámbito económico, por la economía informal, así como en el urbanismo, por el desorden generado por la espontaneidad de dichos asentamientos. La ciudad así entendida se asimila con focos de violencia y frustración ciudadana, alejada de la bucólica visión de la vida rural. En Túnez, por ejemplo, las familias que han emigrado del campo, se convierten en familias excluidas, y se instalan tanto en el centro de la ciudad, como en los alrededores de la misma.

Este proceso de concentración en los países del Magreb está estrechamente relacionado con los servicios, y la voluntad de las poblaciones de beneficiarse tanto de los equipamientos, como agua corriente, electricidad y gas, como de los servicios mercantiles y no mercantiles, comercios y negocios y sanidad y educación, respectivamente. Los centros son los primeros en adquirir los servicios y en función de los mismos las sociedades tienden a concentrarse.

La actual situación de crecimiento desordenado de las ciudades magrebíes ha generado una serie de problemas a los que las sociedades deben hacer frente. No se trata solo de una dimensión espacial, sino que la gestión urbana debe dar respuesta a ciertos desafíos.

Atendiendo al ámbito económico, es la economía sumergida e informal el principal problema. Sin embargo, será la creación de empleo, debido al gran incremento de la población activa, de un $47 \%$ hasta el año 2050 , el principal reto a afrontar por los países magrebíes ${ }^{42}$.

\footnotetext{
${ }^{41}$ Toma su nombre de los años 30, cuando en Túnez, los inmigrantes rurales que llegaban a la Medina, se instalaron en 'fonduks' (fondas), 'ukalas' (posadas) y cementerios situados dentro y fuera del recinto amurallado. Las familias campesinas se instalaron en las viviendas tradicionales abandonadas. Estas casas se alquilaron habitación por habitación y recibieron la denominación de "ukalas", que hasta entonces había designado exclusivamente a los establecimientos que arrendaban cuartos por día o semana a trabajadores solteros. El fenómeno de la "ukalización" no sólo afectó a las casas tradicionales, sino a todo tipo de construcciones: palacios, mansiones, madrasas y edificios religiosos, etc. En cada habitación vivía una familia, en esas condiciones, los inquilinos compartían letrinas, fuentes y cocinas.

Las "ukalas" planteaban problemas de insalubridad, promiscuidad, delincuencia y hacinamiento de las personas en condiciones inhumanas y, por consiguiente, constituían un fenómeno muy preocupante y grave en el plano social, que contribuyeron a la pauperización de la Medina; Akrout-Yaiche, op. cit., nota 37.

42 Marín I.: "Vulnerabilidades socioeconómicas en el Magreb: el reto del empleo", Real Instituto Elcano, ARI, $\mathrm{n}^{\circ}$ 43 (Abril 2005), en http://www.realinstitutoelcano.org/analisis/715/IvanMartin.pdf.
} 
Tabla 4. Indicadores población de los suburbios:

\begin{tabular}{|c|c|c|c|c|}
\hline$\%$ & \multicolumn{2}{|c|}{ Acceso agua potable } & \multicolumn{2}{c|}{ Acceso Sanidad } \\
\hline ANOS & 2001 & 2008 & 2001 & 2008 \\
\hline Argelia & 98 & 85 & 90 & 98 \\
\hline Marruecos & 100 & 98 & 94 & 83 \\
\hline Túnez & 100 & 99 & 96 & 96 \\
\hline
\end{tabular}

Fuente: Naciones Unidas ${ }^{43}$

Uno de los retos a nivel nacional en los países del Magreb es la gestión de los recursos, y de forma muy especial la gestión del agua, debido al desarrollo demográfico y a la urbanización. Marruecos, Argelia y Túnez han adoptado políticas en este sentido, destinadas a la gestión eficaz de los recursos relacionados con el medio ambiente ${ }^{44}$.

Una política de obras públicas destinada a subsanar los errores de la urbanización descontrolada es una oportunidad para la región. En este sentido, la creación de infraestructuras tales como viviendas o saneamientos, así como infraestructuras productivas, como el transporte y las comunicaciones, pueden favorecer y mejorar la gestión urbana, a la vez que contribuyen a la generación de empleo y atraen inversión extranjera.

En cuanto a los servicios básicos, la falta de infraestructuras y la masificación de la demanda de sanidad y educación principalmente, suponen un problema añadido en las áreas urbanas. En cuanto al ámbito de la educación la problemática es doble: por un lado, la ausencia de personal cualificado para atender a la demanda actual, y, por otro, la batalla de la escolarización de la mujer.

La incidencia de la pobreza, entendida como el porcentaje de la población con unos ingresos diarios inferiores a 2 dólares, que se había conseguido reducir del $21 \%$ al $13 \%$ entre 1984 y 1992, repuntó hasta el 19\% en el año 2000 (5,3 millones de pobres frente a los 3,4 millones que había en 1990). La pobreza urbana y su plasmación urbanística es una consecuencia de los procesos de urbanización, por lo que se convierte en una tarea ineludible dotar a esa población urbana marginal de capacidades y niveles de vida tanto socialmente aceptables, como sostenibles. La menor incidencia de la pobreza en los países del Magreb en comparación con otros países en desarrollo, se debe a variables institucionales y culturales. La red de solidaridad existente en la propia religión musulmana y sus valores familiares, proporcionan una red de seguridad social no gubernamental instrumentalizada ${ }^{45}$.

Otro de los desafíos de las sociedades urbanas es la creación de una sociedad civil fuerte, que lleve a cabo procesos de desarrollo autónomo. En este campo la cooperación

\footnotetext{
${ }^{43}$ Para 2001 en: "Afghanistan", op. cit., y para 2008 en http://www.unhabitat.org/downloads/docs/GRHS2011_Full.pdf.

${ }^{44}$ Para más información: Mohammed, Benblidia: "Problemas del agua en el Magreb", Revista Política Exterior, $\mathrm{n}^{\mathrm{o}} 24$ (2003), pp.91-100.

${ }^{45}$ Ver Youssef, Courbage et al. (2009): Población y desarrollo en el Mediterráneo; transiciones demográficas y desigualdades socioeconómicas, Barcelona, Icaria antrazyt/IEMed Mediterráneo, Primer Foro Mediterráneo de Población. Barcelona (11 y 12 de diciembre de 2007).
} 
internacional viene desempeñando una labor destacable, tanto en las zonas urbanas como rurales en los tres países.

\section{Provecciones de urbanización en el Magreb}

La población urbana del Magreb se ha triplicado desde los procesos de independencia, y, a pesar del descenso de la natalidad, la tendencia en un horizonte cercano y medio es de una sociedad joven, o adulta joven. La actual estructura demográfica plantea, como ya se ha indicado, problemas específicos, principalmente en los centros urbanos.

En los países árabes las tasas de crecimiento urbano se mantendrán previsiblemente por encima de las tasas de crecimiento de la población. Así el crecimiento urbano ha sido resultado del 'trasvase' de personas de las zonas rurales a las urbanas, así como altas tasas de fecundidad y reducción de las tasas de mortalidad ${ }^{46}$.

Para el año 2050 Argelia, Marruecos y Túnez tienen unas previsiones para su población urbana de cerca del $90 \%$, superior al $72 \%$ y rozando el $80 \%$ respectivamente ${ }^{47}$. En 2050 Argelia tendrá cerca de 48 millones de habitantes en áreas urbanas, en Marruecos se estima que la población urbana alcanzará los 40 millones, mientras que en Túnez, superarán los 12 millones de habitantes en zonas urbanas.

Gráfico 8. Tasa de crecimiento de la urbanización. 2011 - 2025:

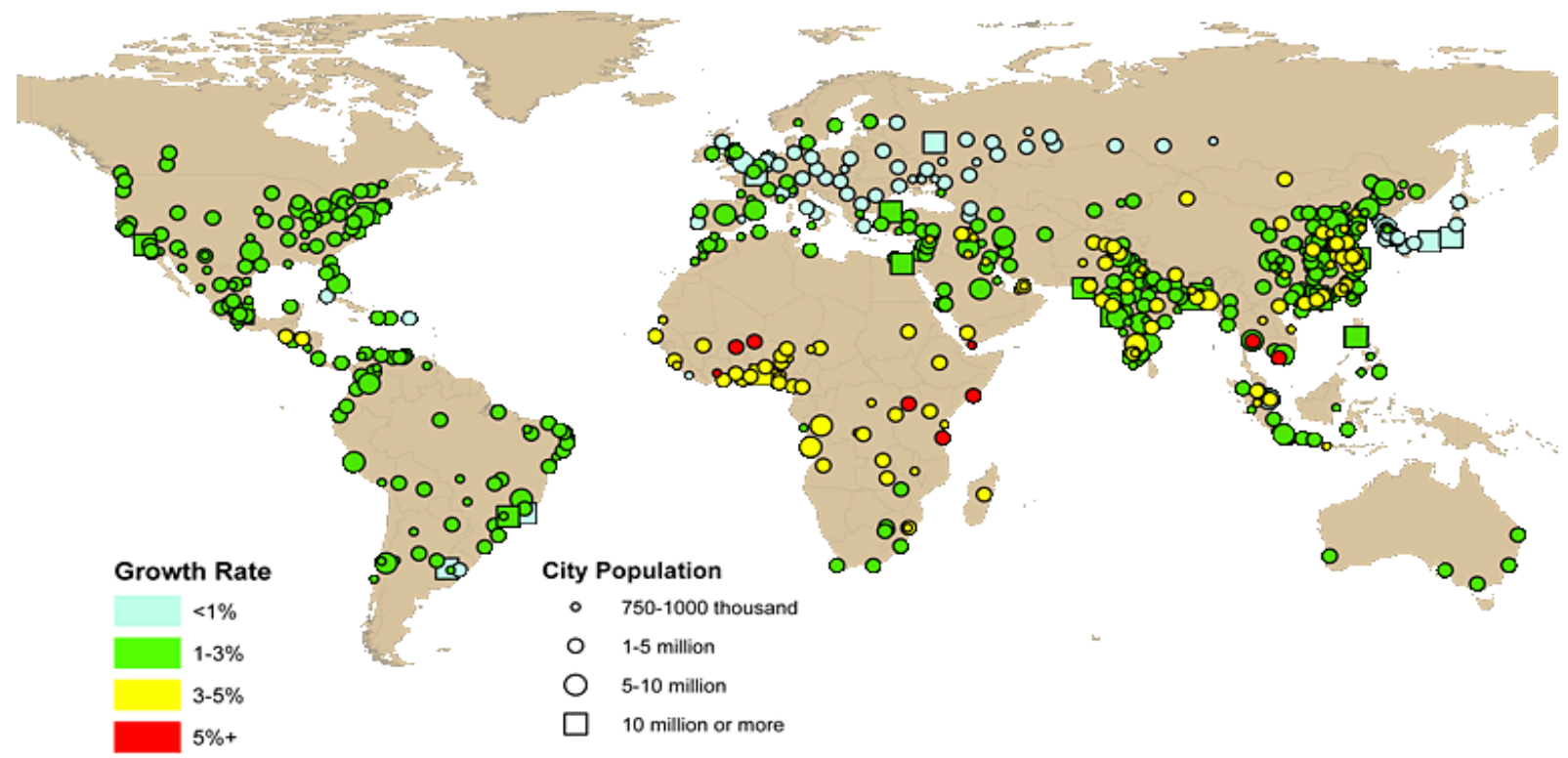

Fuente: Naciones Unidas ${ }^{48}$

\footnotetext{
${ }^{46}$ Datos 2001, estimaciones: UN-HABITAT, en: http://www.unhabitat.org/content.asp?typeid=19\&catid=555\&cid=5374.

47 "World Urbanization Prospects", op. cit.

48 "World Urbanization Prospects", op. cit.
} 
Las tasas anuales de crecimiento de las aglomeraciones urbanas en el período 1970-2011 fueron más altas que las tasas de crecimiento proyectadas para el futuro (2011 a 2025). El proceso de urbanización se reducirá, ya que muchos de los focos urbanos han alcanzado una población de 1 millón o más o se han convertido en megaciudades que superan los 10 millones de habitantes.

En este sentido, Brauch identifica una serie de retos en la región como consecuencia directa de la situación poblacional en el Magreb, así como las previsiones de su crecimiento ${ }^{49}$ : diferentes niveles de población entre los Estados del norte y del sur de la ribera mediterránea; el impacto que el cambio climático tendrá a nivel regional, con las predicciones sobre el incremento de la temperatura global, y el aumento del nivel del mar; la escasez de agua, tanto para el consumo humano como para el uso agrícola; la ausencia de un sistema de autoabastecimiento de la producción alimentaria y el fuerte incremento de la demanda de cereales; la progresiva desertificación a todos los niveles en todos los países del Mediterráneo; y el progresivo proceso de urbanización y polución en las principales ciudades, donde en algunos casos se superarán en el año 2015 los 12,5 millones de personas en Estambul o los 14.5 millones en El Cairo. En particular, en relación con el sistema alimentario, si la población continúa urbanizándose y creciendo de forma regular, los recursos de la renta de exportación, comienzan a diluirse progresivamente como resultado de la caída de los precios de las materias primas en el mercado internacional ${ }^{50}$. El incremento de la población contribuye directamente al incremento de la demanda de agua y comida ${ }^{51}$.

En los próximos años esta región va a ser clave a la hora de abordar su evolución demográfica, en particular un incremento de la población activa de aproximadamente 750.000 personas al año, y en el que los procesos de inmigración afectan de forma directa a sus vecinos europeos. La demografía magrebí supone por tanto un desafío para los tres Estados analizados, así como uno de los factores principales de riesgo en el entorno.

\section{Conclusiones}

El Magreb es una región que aporta actualmente la cuarta parte de la riqueza del continente africano, y posee una economía y una sociedad en proceso de actualización. En las últimas décadas el amplio proceso en la explotación de minerales e hidrocarburos ha generado cierto bienestar y desarrollo económico basado en la industrialización en países como Marruecos, Argelia, Túnez o Libia. La industria proporciona, según países, entre un tercio y algo más de la mitad del PNB y ocupa entre el $10 \%$ y el $30 \%$ de la población activa. La agricultura es uno de los sectores económicos clave, puesto que se encuentra pendiente de una modernización que incremente su productividad. Actualmente la contribución del sector agrícola a la producción nacional no se corresponde con la población activa que emplea: en Marruecos supone un 14\% del PIB nacional, absorbiendo más del $44 \%$ del empleo; en Túnez significa un $2 \%$ de la población activa, mientras que representa un 12\% del PIB; en Argelia supone un 8\% del PIB, y emplea al $12 \%$ de la población ${ }^{52}$.

\footnotetext{
49 Brauch Hans Günter (2001): “The Mediterranean Space and Boundaries”, en Antonio, Marquina, Hans Günter, Brauch (eds.) (2001): The Mediterranean Space ant its Borders. Geography, Politics, Economics and Environment, Madrid, Collection Strademed 14, UNISCI - Mosbach: AFES-PRESS, pp. 25-58.

${ }^{50}$ Abdelhamid, Bencharif y Raúl, Green: "Transición económica y nueva racionalidad productiva: el sector alimentario en el Magreb", revista agroalimentaria, vol. 2, no. 3 (1996).

${ }^{51}$ Brauch, "The Mediterranean Space...", op. cit. nota 46.

${ }^{52}$ Fargues, op. cit., nota 34.
} 
Este incipiente bienestar económico debe traducirse en garantizar la seguridad humana, entendida como que los individuos y comunidades constituidas tengan asegurado el mantenimiento o el restablecimiento de un entorno habitable para las generaciones presentes y futuras. Esta seguridad implica también dar respuesta a las necesidades humanas fundamentales especialmente en materia de nutrición, educación, vivienda y salud pública.

El aumento de la población urbana y las proyecciones sobre incremento de la urbanización en el Magreb central determinarán no sólo la evolución de ciudades y sociedades sino también inducirán una creciente demanda de alimentos por estas agrupaciones humanas que no los producen.

A pesar de que la transición demográfica va a ralentizar el crecimiento de la población, la presión sobre el mercado de trabajo, sobre los servicios públicos y sobre los recursos (alimentos, agua, etc.) va a seguir patente a corto y medio plazo en estos Estados. 